\title{
A Mobile Social and Communication Tool for Autism
}

\author{
https://doi.org/10.3991/ijet.v14i19.10887 \\ Heba Mohammad ( ${ }^{\bowtie}$, Fadi Abu-Amara \\ Higher College of Technology, Abu Dhabi, UAE \\ hmohammadehct.ac.ae
}

\begin{abstract}
Autism is a complex neurobiological disorder that is prevalence worldwide. Most autistic children have weak communication and social skills. This research aims to develop and test a mobile application, named MyVoice, which supports local autistic children. The proposed design and features are discussed, and a prototype is evaluated and tested by two therapists and an autistic child. Experimental results indicate positive feedback in terms of ease of use, aesthetic, and simplicity. Parents of the autistic child are satisfied with different features such as the alert notification. Results also indicate that autistic children need about one week to easily interact with MyVoice.
\end{abstract}

Keywords-Assistive technologies, autism, social skills, learning, mobile application

\section{Introduction}

The prevalence of autism had been recognized to be 1 in every 68 children worldwide [1]. In the UAE, the rate is 1 in every 146 births [2]. It is predicted that these levels will increase in the coming years.

Autism is defined as a complex neurobiological disorder. Autism forms a part of autism spectrum disorders (ASD) [3]. Autistic therapists agreed that autism may appear during the first three years of a child's life where it affects the normal brain functions which consequently affects the development of communication and interaction skills. The way an autistic child interacts with other individuals is very different in comparison with that of normal children. In some cases, autistic children are not interested in communication and interaction with other people at all. Further, autistic children rarely establish eye contact with others. Also, they face difficulty in an understanding feeling of other people or in communicating their own feelings. In addition, autistic children don't like to be touched like other children do. Also, they found loud voices very unpleasant [4]. All these behaviors should be taken into consideration when any interaction is established with an autistic child. In terms of speech and conversation skills, many autistic children cannot properly develop these skills. Approximately, 50\% of children with ASD do not speak at all [5]. Although there is no known biological marker for autism, it can be diagnosed by three main criteria that are behaviorally defined as [6]: Lack of social interaction

- Lack of social communication 
- A restricted range of interests and behaviors

Therefore, therapists use different methods to improve the interaction skills of autistic children. One of the effective methods that had been highly recommended and used to overcome speech and communication problems with autistic children is known as the Augmentative and Alternative Communication [7].

The augmentative and alternative communication (AAC) method uses communication approaches to replace or supplement language, whether spoken or written, of impairment persons. The AAC includes various forms of communication that are used by people with different language problems to express themselves and share their needs. There are other augmentative solutions, like symbol communication boards, pictures, and electronic devices, available to help people with different impairments to communicate with others [8].

In this paper, a mobile application is developed to be used by autistic children to interact with others and communicate their feelings and needs. Autistic therapists and parents will evaluate the performance and features of the proposed application.

The rest of this paper is organized as follows. Section II explores the previous research in this field. Section III illustrates the proposed application design and features while Section IV concludes the paper.

\section{Literature Review}

In this section, different methods proposed in the literature to address the communication and social skills of autistic children are explored.

\subsection{Augmentative and alternative communication}

There are two main types of AAC. The Unaided Communication System does not provide voice output and does not require any electronic hardware. It is like sign language and traditional communication boards. On the other hand, the Aided Communication System uses electronic hardware but may or may not provide voice output. Generally speaking, the aided communication system is more popular [8].

The AAC aided communication system can support augmented input, output, or input and output strategies. In the AAC with input, autistic children can understand other expressions and increase and support language comprehension. On the other hand, the AAC with input and output is designed to support both comprehension and production. The AAC with output is used by autistic children to communicate their feelings to others [8]. There are different ACC methods such as schedules; visual symbols for choice making, aided language stimulation, system augmenting language, visual-spatial symbols, and picture exchange communication system (PECS); to list a few [9-13].

For instance, the Picture Exchange Communication System teaches children with autism the use of visual-graphic symbols for communication [11]. This approach had 
been known for its effectiveness in improving autistic children communication skills [15-16].

Autistic children can communicate in four different abilities according to communication competence. Based on research related to children with autism, each ability has the following communication skills:

Generalized ability: When a child learns a new skill and is able to perform in different settings.

Spontaneous ability: It's basically teaching an autistic child spontaneous response that is defined as specific verbal utterances. For example, a child says "bless you" following a discriminative stimulus that did not involve explicit vocal directives (e.g., adult sneeze). In fact, children with autism do not use spontaneous communication when they are in their early stage of learning [17].

Joint attention ability: Developing certain skills that involve sharing attention with others; by teaching a child to do one thing in different manners. For example, autistic children can point at objects and people to share their interests, bring and show objects to others, and coordinate looking between objects and people $[16,17]$.

Maintenance ability: Refers to ongoing use of skills over time. It's related to evaluate the ability of autistic children to use and improve their communication competence through continuous interaction and use over a period [17].

\subsection{The use of technology with autistic children}

Technology, in general, is used to improve the quality of a person's life and it's been used, especially, to help people with difficulties to make their life easier. Nowadays, there are different technologies used to help children with autism in different aspects. The technology can be used to aid autistic children in the overall understanding of their environment, improve their expressive communication skills, social interaction skills, attention skill, organization skill, motivation skill, academic skills, selfhelp skills, and overall independent daily functioning skills [16, 17]. For instance, video can help specialists in early detection of autism since this technique made it easier for parents to film their children from anywhere to observe them and then films are evaluated by a specialist [18]. Currently, video is used to teach children with autism the basic communication skills by showing them videotaped conversations. Video technology has proven that it is useful as a tool for modeling appropriate behavior, providing feedback, creating discrimination opportunities for an autistic child's own behavior, and as a way for presenting basic instructions that many children can be evolved in [19]. In summary, children with autism can understand visual representations better than auditory ones [19, 20].

Another example of using technology with autistic children is through using different types of assistive technologies. Assistive technology can be used to maintain or improve the functional capabilities of people with disabilities [21]. Assistive technology utilizes pictures that children can use to make a request, or it can utilize speech generating devices [21, 22].

An example of assistive technology includes mind reading devices. These devices are used to teach autistic children emotions and face expressions. They work as a 
guide to children's mental state by providing images or videos of people expressing different emotions [18]. These devices are very helpful in places like homes and schools to help autistic children in expressing their feelings and emotions.

Another example of assistive technology includes virtual reality (VR). VR can help an autistic child to acquire and practice new skills based on real situations where he/she had a chance to transfer the learned skills back to real life. For example, autistic children can learn how to safely cross the street. VR uses repeated practices in natural settings to teach a new skill and it offers an adjective control over the environment [23].

One of the promising areas technology use these days that opens new areas for autistic children is the use of tablet devices as a communication method between children and their environments. Tablets are cheaper, faster, more versatile, more userfriendly, more portable, more engaging, and infinitely suitable for autistic children in comparison with other technologies [24].

\subsection{Related work}

With the constant increase in ASD cases and the prevalence of awareness of autism syndromes, there is a need for an application that can be used to aid treatment and diagnoses of autism syndrome. A search on Apple and Google play stores indicates different applications related to autism with different categories ranging from entrainment to educational. Most of the applications are built based on PECS method concepts and used to enhance the communication skills of autistic people. However, there is a need for an application that can be customized to the UAE environment. This section explores common applications developed for autistic children.

- Tap to talk: A user can create a personal album according to the teacher and the mother's need. Its library contains more than 20,000 pictures. A child taps on a picture to hear audio. This application lacks a voice recording feature directly from the iPad. Also, the user should connect the iPad to the computer each time to download or delete a picture or audio. Further, it contains some material inappropriate with the UAE culture. Finally, it does not support the Arabic language.

- Proloquo2Go: Contains an updated vocabulary, text to speech voices and automatic conjugation. It's customizable where a parent or teacher can add a new picture from the tablet itself. However, the interface a little bit confusing to the child and it is not well organized. Also, it is more suitable for adults and high-level children. If a child keeps tapping on a picture, it will be added several times. Further, it contains some material inappropriate with the UAE culture. Finally, it does not support the Arabic language.

- Icommunicate: It allows a user to design personal visual schedules, stories, communication board, and speed card. It also allows recording user's voice to be associated with a picture or use existing different languages. A user can use personal pictures that are stored in the tablet's library or take pictures directly from the camera. The interface a little bit confusing to the child and it is not well organized. Fur- 
ther, it contains some material inappropriate with the UAE culture. Finally, it does not support the Arabic language.

\section{Proposed MyVoice Application Design and Features}

As discussed in the previous section, technology can be used to improve the social and communication skills of autistic children. Therefore, this work aims at developing a mobile application, named MyVoice, to be used in improving the communication skills of Emirati autistic children. The proposed therapeutic and affordable tool for autistic children is intended to teach words and how to communicate feelings and needs in a convenient approach.

\subsection{Proposed myoice application features}

My Voice is an iOS-based application designed to be installed on iPad tablet for UAE autistic children to enable them to communicate by using their native Arabic language. The application contains a collection of albums in a library in which a child taps on a picture to generate spoken sentences and phrases, such as "I want ice cream". For example, a child taps on the picture that represents him/her then taps a picture that describes an action (want) and then taps a picture that describes the ice cream. In the end, the selected pictures create a sentence that describes what the child wants to say as shown in Figure 1-A.

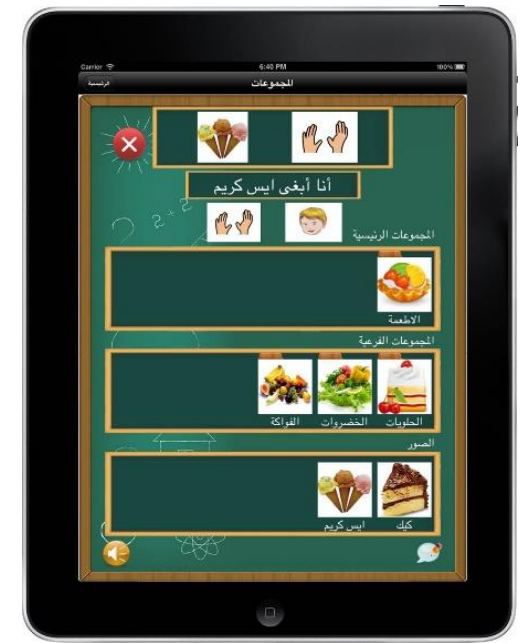

Fig. 1. (a) Example of "I want ice cream" spoken sentence generation and

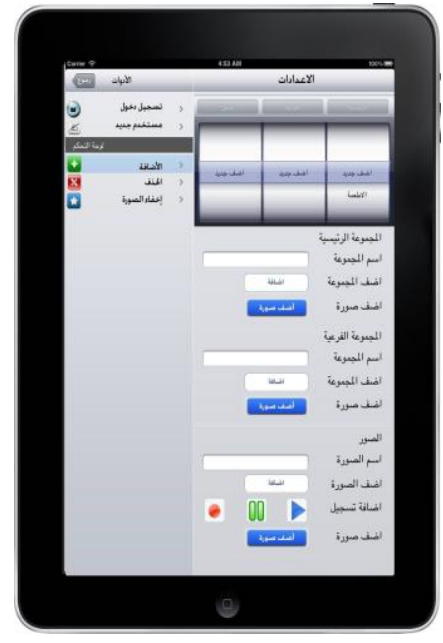

(b) Adding picture to a category 
The proposed application allows parents and therapists to create categories, add pictures within a category, and record an audio per picture. Also, the application can alert a caregiver about the feelings of the autistic child based on the sentences structured by him/her. For example, if a child creates a sentence that describes his/her emotional state as "sad", "hungry", "scared" or "in pain", a notification message will be automatically sent to the caregiver about the child's emotional status. This feature could be set on if the caregiver was in a far distance from the child. As far as we know, such a feature is not provided in similar applications related to autistic children.

To use MyVoice, the user must create an account. Figure 1-B shows the interface of adding a picture to a category. It allows the user to select category name, create a new category name, add a subcategory to a current category, determine a name for the picture, record audio per picture, delete a category, delete a picture, and/or delete audio.

The MyVoice application is designed to support two actors; caregiver and autistic child. The caregiver can create an account as a caregiver and then can create another account for the autistic child. The caregiver can create a category, add pictures to a category, and record audio associated with each picture. Also, the caregiver can hide a category or picture and can mute audio for a specific child as shown in Figure 2. In this case, if a child wants to say, "I want an apple", he/she will not be apple to choose the apple picture because it is hidden and inactive. On the other hand, an autistic child can build a sentence by tapping twice on a picture, then the picture will be added to the sentence bar and audio will be played with each tap. However, if the child continues tapping for five times, a notification message will be automatically sent to the registered parent mobile phone in order to help the child.

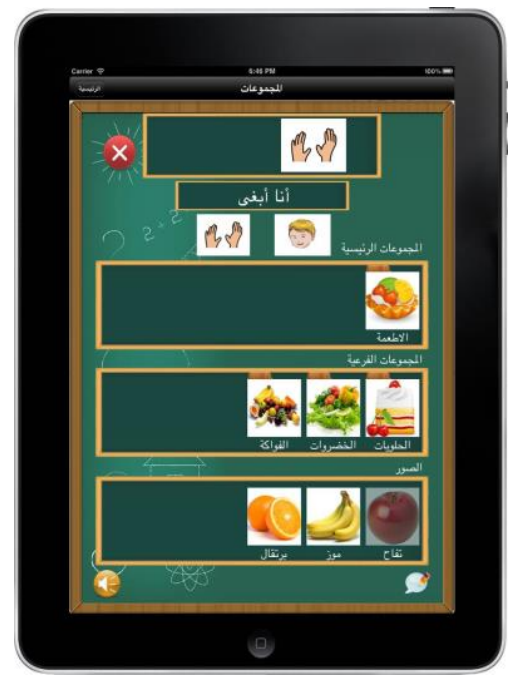

Fig. 2. Fruit category with apple picture disabled 


\subsection{Experimental results and evaluation}

The MyVoice application is tested and evaluated by two autistic therapists at local autism centers and parents. Both therapists were involved in teaching autistic children. The therapists evaluated the proposed application with positive feedback on the application's ease of use, aesthetic, and simplicity. Both therapists highly recommend MyVoice to be used in local autism centers.

After testing the application by the two therapists, an experiment had been conducted. The adopted methodology was single-subject research design. One child had been selected to use the application and do the test. The therapists helped in choosing the child and to make sure that the child does not know the content of the application but she is familiar with pecs. The child has to do a test for the first time and the score was considered as a reference value to measure how the application will help in improving the child learning. Then the child had to practice on the application and to do the test again.

On the other hand, one autistic child used MyVoice application for two months. The child did not show an acceptable level of interaction with MyVoice application during the first week. However, an improvement with time in the interaction level had been noticed by the therapist. The child was able to express his needs after one week of interaction. This quick adaption from the child is due to the comfortability of the child with the PECS approach before introducing the application.

Parents of the corresponding autistic child were very satisfied with MyVoice during the experimentation period. The parents liked the features listed in Section 3.1. Also, they liked the alert notification feature since parents may not be in a close distance with their child. Also, the appropriateness of the application to the UAE culture and the Arabic language support made MyVoice the preferred parents' autistic application.

\section{Conclusion and Future Work}

In this work, MyVoice application was developed as a need raised by local autism centers and parents who were looking for an easier, cheaper, and convenient tool to improve their autistic children communication and social skills. The application is considered as an attempt to fill the need for an autism educational application in native Arabic language and to be suitable for the local culture. Experimental results indicate that parents and therapists can efficiently and conveniently use MyVoice application to communicate and educate their autistic children. Results also indicate that it takes about one week for autistic children to easily interact with MyVoice. It is preferable that targeted autistic children have experience with PECS approach.

As future work, a two-year experiment will be conducted to evaluate how effective is the application in the educational process. Also, per the therapist' recommendations, more games and fun elements will be added to the application to make it more attractive to children. Finally, multi-factor authentication will be integrated to improve the application's security. 


\section{$5 \quad$ References}

[1] Pandey, A.K. and Abhishek, P. (2018). An Update on oral health management in the world of autism- a review. Austin Pediatr. 5 (1): 1-5.

[2] Al Abbady, K., Hessian, H.Y., and Alaam, M.W. (2017). Prevalence, trend, determinants and prediction of autism spectrum disorders among Dubai population. Diagnostic Approach and Management Contexts. Journal of Pediatr Neonatal Care. 7 (1), doi: 0.15406/jpnc.2017.07.00278. https://doi.org/10.15406/jpnc.2017.07.00278

[3] American Psychiatric Association. 2013. Diagnostic and statistical manual of mental disorders. 5th ed. Arlington. VA: American Psychiatric Association. https://doi.org/10.1176/ appi.books. 9780890425596

[4] Fátim, M., Doval, G., José, M., Carballo, P., Manuel, J., and Jeremías, V. (2010). TICTAC: information and communication technologies for augmentative communication boards. IEEE EDUCON 2010 Conference. Madrid, pp. 1783-1787.https://doi.org/10. 1109/EDUCON.2010.5492419

[5] Rudy, L.J. 2018. Autism and the picture exchange communication system.

[6] Frost, L. and Bondy, A. (2013). A clear picture: the use and benefits of PECS. available at: shttp://www.pecs.com/webcasts/ClearPictureHandout.pdf.

[7] Collet-Klingenberg, L. (2008). Overview of picture exchange communication system (PECS) for children and youth with autism spectrum disorders. Madison, WI: National. pp. $1-3$.

[8] Beukelman, D. and Mirenda, P. (2013). Augmentative and alternative communication: supporting children and adults with complex communication needs. 4th ed., Baltimore, MD: Paul Brookes Publishing Co.

[9] Agius, M.M. and Vance, M. (2016). A comparison of pecs and ipad to teach requesting to pre-schoolers with autistic spectrum disorders. AAC: Augmentative \& Alternative Communication. 32 (1): 58-68, March 2016. https://doi.org/10.3109/07434618.2015.1108363

[10] Millar, D.C., Light, J.C., and Schlosser, R.W. (2006). The impact of augmentative and alternative communication intervention on the speech production of individuals with developmental disabilities: a research review. Journal of Speech, Language, and Hearing Research. 49 (2): 248-264. https://doi.org/10.1044/1092-4388(2006/021)

[11] Mirenda, P. (2001). Autism, augmentative communication, and assistive technology what do we really know?. Focus on Autism and Other Developmental Disabilities. 16 (3): 141151. https://doi.org/10.1177/108835760101600302

[12] Mirenda, P. (2003). Toward a functional and augmentative and alternative communication for students with autism: manual signs, graphic symbols, and voice output communication aids. Learning, Speech, and Hearing Services in Schools. 34 (3): 203-216. https://doi.org/10.1044/0161-1461(2003/017)

[13] Thiemann-Bourque, K., Brady, N., McGuff, S., Stump, K., and Naylor, A. (2016). Picture exchange communication system and pals: a peer-mediated augmentative and alternative communication intervention for minimally verbal preschoolers with autism. Journal of Speech, Language and Hearing Research. 59 (5): 1133-1145. https://doi.org/10.10 44/2016_JSLHR-L-15-0313

[14] Nunes, D. and Walter, C. (2018). AAC and autism in Brazil: a descriptive review. International Journal of Disability, Development, and Education. pp. 1-17. https://doi.org/10. 1080/1034912X.2018.1515424

[15] Gobrial, E. McAnelly, S., and Shannon, P. (2018). Education of children and young people with autistic spectrum disorders in Egypt. British Journal of Learning Disabilities. 4: 2934. https://doi.org/10.1111/bld.12250 
[16] Ostryn, C. and SA, P. (2009). Review and analysis of the picture exchange communication system (PECS) for individuals with autism spectrum using a paradigm of communication competence. Research \& Practice for Persons with Severe Disabilities 2009. 33: 13-24. https://doi.org/10.2511/rpsd.33.1-2.13

[17] Michel, P. (2004). The use of technology in the study, diagnosis and treatment of autism. Final term paper for CSC350: Autism and Associated Developmental Disorders. pp. 1-26.

[18] Charlop, M.H., Lang, R., and Rispoli, M. (2018). Lights, camera, action! teaching play and social skills to children with autism spectrum disorder through video modeling. In book: Play and Social Skills for Children with Autism Spectrum Disorder. Springer, Cham. pp. 71-94. https://doi.org/10.1007/978-3-319-72500-0

[19] NHS Education for Scotland. (2007). Assessment, diagnosis and clinical interventions for children and young people with autism spectrum disorders. Scotland: Scottish Intercollegiate Guidelines Network. pp. 1-60.

[20] Benssassi, E.M., Gomez, J., Boyd, L.E., Hayes, G.R., and Ye, J. (2018). Wearable assistive technologies for autism: opportunities and challenges. IEEE Pervasive Computing. 17 (2): 11-21, April-June 2018. https://doi.org/10.1109/MPRV.2018.022511239

[21] Zorcec, T., Robins, B., and Dautenhahn, K. (2018). Getting engaged: assisted play with a humanoid robot Kaspar for children with severe autism. ICT Innovations 2018. Engineering and Life Sciences. 940: 198-207. https://doi.org/10.1007/978-3-030-00825-3_17

[22] Mesa-Gresa, P., Gil-Gómez, H., Lozano-Quilis, J.A., and Gil-Gómez, J.A. (2018). Effectiveness of virtual reality for children and adolescents with autism spectrum disorder: an evidence-based systematic review. Sensors (Basel). 18 (8): pii: E2486. doi: 10.3390/s18082486. https://doi.org/10.3390/s18082486

[23] Rasche, N. (2013). Design strategy for the development of applications for autism instruction. Open Access Theses. Purdue University.

\section{Authors}

Heba Mohammad is an Assistant Professor at the Computer and Information Sciences Division of the Higher Colleges of Technology, Abu Dhabi, UAE. Heba worked as an Assistant Professor in Saudi Arabia and Jordan. In addition, she worked as a researcher in Italy. Heba received her $\mathrm{Ph} . \mathrm{D}$ in e-business from University of Salento, Italy. Her research focuses on knowledge Discovery and its applications, Knowledge Management e-Business \& e-learning and Emerging Technologies. Email id: hmohammad@hct.ac.ae

Fadi Abu-Amara is an Assistant Professor at the Computer and Information Sciences Division of the Higher Colleges of Technology, Abu Dhabi, UAE. He served as a program chair for five years. He has an active academic carrier with more than fourteen years of teaching and research activities in various reputed institutes and universities. Fadi received his $\mathrm{PhD}$ in Computer Engineering from Western Michigan University (USA) in 2010. His fields of interests include cryptography, cybersecurity, biomedical engineering, feature extraction, socially assistive robotics, and concrete bridge defect detection. He is also actively involved in promoting virtual and augmented reality to undergraduate students. Email: fabuamara@hct.ac.ae

Article submitted 2019-05-18. Resubmitted 2019-06-03. Final acceptance 2019-06-29. Final version published as submitted by the authors 